\title{
A dynamics modeling research for angular contact ball bearing of high-speed spindle under rigid preload
}

\author{
ZhifengLiu ${ }^{1,}$,, Bohua Zhang ${ }^{2, b}$ \\ ${ }^{1}$ School of Beijing University of Technology,Beijing 100124,China; \\ ${ }^{2}$ School of Beijing University of Technology,Beijing 100124,China. \\ a'zf@bjut.edu.cn, b1005126748@qq.com
}

Keywords:angular contact ball bearings; Hertz contact theory; rigid preload; high-speed spindle; dynamic model.

\begin{abstract}
Determining the parameters of angular contact ball bearings under preload is the key issue to improve the accuracy and performance of high-speed spindle. The impact of gyro force and centrifugal force were considered, a static and a dynamic model for angular contact ball bearings under rigid preload were presented. By using Newton iterative method, dynamic parameters of the angular contact bearings were obtained, including eccentricity of contact ellipse, contact angle, contact force, maximum contact stress, contact ellipse radii and stiffness, etc. According to numerical simulation, relationship between the parameters, versus preload and versus rotation speed were given. The results reveal that the stiffness increases as the preload increases appropriately, the contact stress and contact ellipse radii increase as the preload increases excessively. In addition, under rigid preload this model has no bearing softening effect. This method provides valuable theoretical basis for further researches, such as calculating bearingscalorific value, calculating bearings fatigue life and optimizing bearings structure, etc.
\end{abstract}

\section{Introduction}

Angular contact ball bearings are commonly used in high-speed spindles. The performance of angular contact ball bearings effect the static and dynamic performance of high-speed spindles seriously [1]. These bearings require preloading in order to improve the rotational accuracy and sufficient stiffness, and reduce vibration noise, etc. Basically, there are two types of bearing preload: rigid and constant. Constant preload is using helical springs, disc springs, and other preload devices to make the bearings obtain appropriate preload force. Rigid preload is using sleeves and washers to make the inner ring and outer ring in an appropriate distance, so that the bearings have appropriate preload force [2]. In actual manufacturing process, because of lacking accurate modeling and analyzing method, it is a key problem to determine the preload force or preload distance of the bearings. Consequently, it is significant to establish a model of bearings under preload.

There are many studies make researches about bearings in modeling, analyzing, testing fields. Altintas considered gyro force and centrifugal force of rotation parts, and established a model by using the finite element method [3,4]. Lim derived 5 degree of freedom analysis model and stiffness matrix based on Hertz contact theory [5,6]. Royston derived 5 degree of freedom vibration model based on Lim's work. And studied the change of natural frequency and stiffness in different preload force [7]. LigangCai presented a modeling theory of angular contact ball bearings under constant preload, and verified the model through an experiment [8]. Wang Hong-jun introduced bearing softening effect under high speed rotation [9]. Wensing calculated elastic deformation according to the generalized shape function, and used software ANSYS to verify the result [10]. BaominWang analyzed mechanical properties of bearings by using Pseudo dynamic method [11]. Ali investigated the stiffness of bearings under different preload force and rotation speed through hammer test [12]. TaipingHuang used eccentric mass excitation method to obtain the equivalent stiffness of the bearings [13].

Few investigations have focused on the modeling method of angular contact ball bearings under rigid preload. This paper based on Hertz contact theory, and considered the impact of gyro force 
and centrifugal force. This paper presented a new model of angular contact ball bearings under rigid preload, including contact area model, preload model in static state, and preload model in rotating state. According to Newton iterative method, important parameters of the model in versus preload force and versus rotation speed were obtained (such as eccentricity of contact ellipse, contact angle, contact force, maximum contact stress, contact ellipse radii and stiffness). This method provides valuable theoretical basis for further optimizing research, fatigue life research, etc.

\section{Dynamic model of angular contact ball bearings}

This paper based on Hertz contact theory, established contact area model, and obtained the parameter equations of eccentricity of contact ellipse. Then, preload model in static state was established and force balance equations was obtained. According to the equations, the actual contact angle $a^{\prime}$ can be solved. And then, the impact of gyro force and centrifugal force were considered, preload model in rotating state was established, and nonlinear equations about eccentricity of contact ellipse $\left(\mathrm{e}_{\mathrm{i}}, \mathrm{e}_{\mathrm{o}}\right)$, contact angle $\left(\theta_{\mathrm{ik}}, \theta_{\mathrm{ok}}\right)$, contact force $\left(\mathrm{Q}_{\mathrm{ik}}, \mathrm{Q}_{\mathrm{ok}}\right)$ were presented. These parameters can be solved by Newton iterative method. At last, stiffness model was established. Making the previous solutions $\left(\mathrm{e}_{\mathrm{i}}, \mathrm{e}_{\mathrm{o}}, \theta_{\mathrm{ik}}, \theta_{\mathrm{ok}}, \mathrm{Q}_{\mathrm{ik}}, \mathrm{Q}_{\mathrm{ok}}\right)$ into stiffness model, the stiffness of axial direction and radial direction can be given.

Contact area model of angular contact ball bearings. According to theBoussinesq solution and Hertz contact theory, the displacement at a point can be expressed as:

$$
\omega(\mathrm{x}, \mathrm{y})=\frac{1-v^{2}}{\pi E} \iint_{S_{c}^{\prime}} \frac{1-v^{2}}{\pi \mathrm{E}} \frac{p_{0}}{\sqrt{(x-x)^{2}+\left(y-y^{\prime}\right)^{2}}} d x^{\prime} d y^{\prime}
$$

Here, $p\left(x^{\prime}, y^{\prime}\right)$ is the distribution pressure, $p_{0}$ is the maximum stress on the center of the contact ellipse,the elliptical contact area has semi-major axis of length a and semi-minor axis of lengthb,and the eccentricity e is given by: $e^{2}=1-(b / a)^{2}, b<a$.

After coordinate transformation:

$\omega=\frac{1-v^{2}}{E}\left(L-M x^{2}-N y^{2}\right)(2)$

Using:

$\mathrm{L}=P_{0} b K(e)(3)$

$\mathrm{M}=\frac{b P_{0}}{a^{2} e^{2}}(K(e)-E(e))(4)$

$\mathrm{N}=\frac{b P_{0}}{a^{2} e^{2}}\left(\frac{a^{2}}{b^{2}} E(e)-K(e)\right)(5)$

By integrating the distributed pressure on the contact area, the external loading $Q$ can be given:

$\iint_{S_{c}^{\prime}} P_{0} \sqrt{1-(x / a)^{2}-(x / b)^{2}} d x d y=2 / 3 \pi \mathrm{ab} P_{0}=Q(6)$

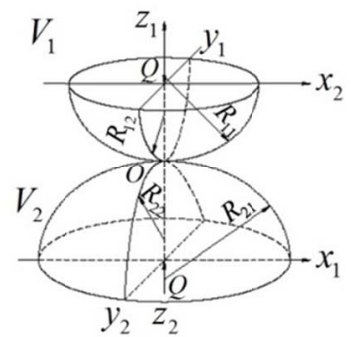

Fig. 1 Point contact diagram

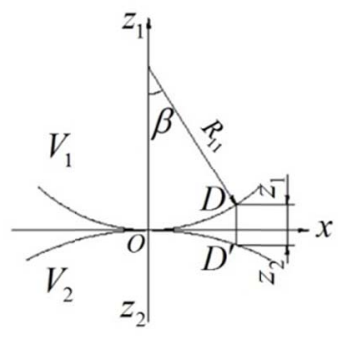

Fig.2 Contact surface distance 


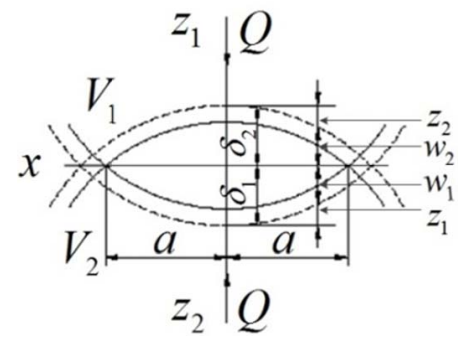

Fig.3 Deformation coordinatesdiagramdiagram

The point $\mathrm{O}$ is the contact zone between the two elastic bodies $\mathrm{V}_{1}$ and $\mathrm{V}_{2}$ before the load is applied, $\mathrm{R}_{11}, \mathrm{R}_{12}, \mathrm{R}_{21}$, and $\mathrm{R}_{22}$ are the main radii of curvature, as shown in Fig 1. DD' is the distance between points $\mathrm{D}\left(\mathrm{x}_{1}, \mathrm{y}_{1}\right)$ and $\mathrm{D}^{\prime}\left(\mathrm{x}_{2} \mathrm{y}_{2}\right)$ as shown in Fig 2.which can be expressed as:

$$
\mathrm{z}=z_{1}+z_{2}=\frac{1}{2}\left(\frac{x_{1}^{2}}{R_{11}}+\frac{y_{1}^{2}}{R_{12}}+\frac{x_{2}^{2}}{R_{21}}+\frac{y_{2}^{2}}{R_{22}}\right)(7)
$$

For an angular contact ball bearing, the rolling body has a spherically symmetric structure, so the rolling element and its contact object's main curvatures are coincident. Hence, $\mathrm{z}$ can be expressed as:

$\mathrm{z}=\frac{1}{2}\left(\frac{1}{R_{11}}+\frac{1}{R_{21}}\right) x^{2}+\frac{1}{2}\left(\frac{1}{R_{12}}+\frac{1}{R_{22}}\right) y^{2}=\mathrm{A} x^{2}+B y^{2}(8)$

Here:

$\mathrm{B}-\mathrm{A}=\frac{1}{2}\left\{\left[\left(\frac{1}{R_{12}}-\frac{1}{R_{21}}\right)+\left(\frac{1}{R_{22}}-\frac{1}{R_{11}}\right)\right]^{2}-4\left(\frac{1}{R_{12}}-\frac{1}{R_{11}}\right)\left(\frac{1}{R_{22}}-\frac{1}{R_{21}}\right) \sin ^{2} \alpha\right\}^{1 / 2}(9)$

$\mathrm{B}+\mathrm{A}=\frac{1}{2}\left(\frac{1}{R_{11}}+\frac{1}{R_{12}}+\frac{1}{R_{21}}+\frac{1}{R_{22}}\right)(10)$

According to the deformation coordinates shown in Fig $3, \omega_{1}(\mathrm{x}, \mathrm{y}), \omega_{2}(\mathrm{x}, \mathrm{y}), z_{1}(\mathrm{x}, \mathrm{y})$, $z_{2}(\mathrm{x}, \mathrm{y})$ are the contact plane displacements, the following equation can be obtained:

$\delta=\delta_{1}+\delta_{2}=\omega_{1}+\omega_{2}+z_{1}+z_{2}(11)$

Combining Eq.(9) to Eq. (11), the equation of eccentricity of contact ellipse e can be obtained:

$\frac{B-A}{B+A}=\mathrm{F}(\rho)=\frac{2\left(1-e^{2}\right)}{e^{2}} \frac{E(e)-K(e)}{E(e)}+1(12)$

Here:

$\mathrm{a}=\left(\frac{2 E(e)}{\pi\left(1-\mathrm{e}^{2}\right)}\right)^{1 / 3}\left(\frac{3 Q}{2 \Sigma \rho E^{\prime}}\right)^{1 / 3}$

$\mathrm{b}=\left[\frac{2 \sqrt{1-e^{2}} E(e)}{\pi}\right]^{1 / 3}\left(\frac{3 Q}{2 \Sigma \rho E^{\prime}}\right)^{1 / 3}$

$\delta=\frac{2 K(e)}{\pi}\left(\frac{\left(1-e^{2}\right) \pi}{2 E(e)}\right)^{1 / 3}\left(\frac{3 Q}{2 \Sigma \rho E \prime}\right)^{2 / 3} \frac{\Sigma \rho}{2}(15)$

$\Sigma \rho=\frac{1}{R_{11}}+\frac{1}{R_{12}}+\frac{1}{R_{21}}+\frac{1}{R_{22}}$ (16)

$\frac{1}{E^{\prime}}=\frac{1-v_{1}^{2}}{E_{1}}+\frac{1-v_{2}^{2}}{E_{2}}(17)$

Preload model in static state of angular contact ball bearings. With a preload force acting on the bearing, the bearing contact angle will change, and the inner ring of the bearing will produce an axial displacement. Assuming the bearing is isotropic in the radial direction,there is no radial displacement. In the preloaded case there is just an axial displacement produced in the inner ring. The outer is fixed and the geometric relationships between the inner and outer rings are as shown in Fig4. 


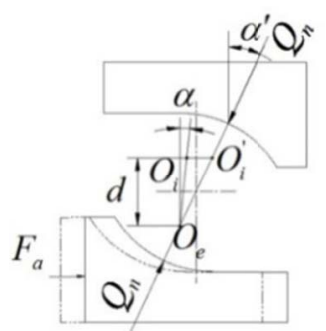

Fig. 4Displacement diagram under static preload

With the axial force $F_{a}$ acting on a bearing, each ball will be subject to the same deformation under the same load. The center of curvature will move from $O_{i}$ to $O_{i}^{\prime}$. $O_{i}$ and $O_{e}$ are the initial groove curvature centers of outer and inner rings respectively; $\alpha$ and $\alpha^{\prime}$ are the initial bearing contact angle and the actual contact angle.

According to the Geometrical relationship, the distance of $O_{i} O_{e}$ and $O_{i}^{\prime} O_{e}$ can be presented:

$O_{i} O_{e}=\left(f_{i}+f_{e}-1\right) D_{w}(18)$

$O_{i}^{\prime} O_{e}=\frac{d}{\cos \alpha \prime}=O_{i} O_{e} \frac{\cos \alpha}{\cos \alpha \prime}(19)$

The difference between $\mathrm{O}_{\mathrm{i}}{ }^{\prime} \mathrm{O}_{\mathrm{e}}$ and $\mathrm{O}_{\mathrm{i}} \mathrm{O}_{\mathrm{e}}$ is $\delta_{\mathrm{n}}$, which is the normal contact deformation:

$\delta_{n}=O_{i}^{\prime} O_{e}-O_{i} O_{e}(20)$

The normal contact load:

$Q_{n}=K_{n} \delta_{n}(21)$

Where, $Z$ is the number of steel balls, $K_{n}$ is the Stiffness coefficient.

$K_{n}=2.1343 * 10^{5}\left(\delta_{i}^{*} \Sigma \rho_{i}^{\frac{1}{3}}+\delta_{o}^{*} \Sigma \rho_{o}^{\frac{1}{3}}\right)(22)$

Force balance equation of the bearings can be presented:

$\mathrm{Z} Q_{n} \sin \alpha^{\prime}=F_{a}(23)$

By substituting Eq.(18) (22) into Eq. (23):

$\frac{F_{a}}{Z K_{n} O_{i} O_{e}{ }^{1.5}}=\sin \alpha^{\prime}\left(\frac{\cos \alpha}{\cos \alpha \prime}-1\right)^{1.5}(24)$

The actual contact angle $a^{\prime}$ can be obtained by solving Eq. (24)

The center of curvature of the steel ball:

$\frac{1}{R_{11}}=\frac{1}{R_{12}}=\frac{2}{D_{w}}(25)$

The center of curvature of the inner ring:

$\frac{1}{R_{i 1}}=-\frac{1}{f_{i} D_{w}}(26)$

$\frac{1}{R_{i 2}}=\frac{2 \cos \alpha}{d_{m}-D_{w} \cos \alpha}(27)$

The center of curvature of the outer ring:

$\frac{1}{R_{01}}=-\frac{1}{f_{e} D_{w}}(28)$

$\frac{1}{R_{o 2}}=\frac{2 \cos \alpha}{d_{m}+D_{w} \cos \alpha}(29)$

By substituting Eq.(25) (29) into Eq. (12), equation $\mathrm{f}_{1}, \mathrm{f}_{2}$ were obtained:

$f_{1}=\frac{2\left(1-e_{i}^{2}\right)}{e_{i}^{2}} \frac{E\left(e_{i}\right)-K\left(e_{i}\right)}{E\left(e_{i}\right)}+1-\frac{B_{i}-A_{i}}{B_{i}+A_{i}}=0$

$f_{2}=\frac{2\left(1-e_{o}^{2}\right)}{e_{o}^{2}} \frac{E\left(e_{o}\right)-K\left(e_{o}\right)}{E\left(e_{o}\right)}+1-\frac{B_{o}-A_{o}}{B_{o}+A_{o}}=0(31)$

Preload model in rotating state of angular contact ball bearings. When the bearing in rotating state, the outer ring is fixed and the inner ring is fixed relative to it as well. With high speed rotation, the centripetal force $\mathrm{F}_{\mathrm{ck}}$ and gyroscopic momentof thebearing $\mathrm{M}_{\mathrm{gk}}$ must be considered, as shown in Fig5. As can be seen in the Fig 6, O' is the initial position of ball center, O', is the final position of ball center, $\mathrm{D}$ is the curvature center of inner ring, and $\mathrm{B}$ is the curvature center of outer ring 


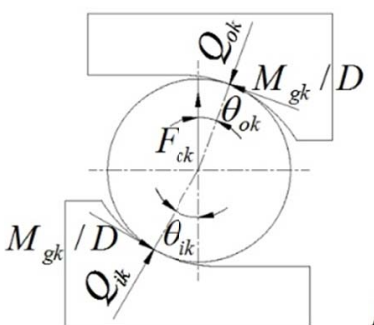

Fig.5 Bearings force relationship

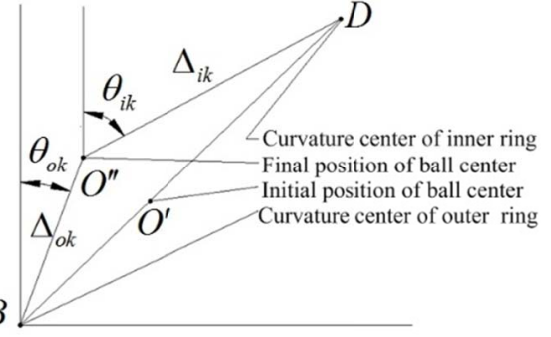

Fig. 6 Displacement diagram

The centripetal force $\mathrm{F}_{\mathrm{ck}}$ and gyroscopic momentof thebearing $\mathrm{M}_{\mathrm{gk}}$ can be presented:

$F_{c k}=\frac{1}{2} m D_{w} \Omega^{2}\left(\frac{\Omega_{E}}{\Omega}\right)^{2}(32)$

$M_{g k}=J_{b} \Omega^{2}\left(\frac{\Omega_{E}}{\Omega}\right)_{k} \sin \alpha_{k}(33)$

The equilibrium equations in the vertical and horizontal directions for the ball can be expressed as:

$f_{3}=F_{c k}+\frac{M_{g k}}{D} \sin \theta_{o k}+Q_{i k} \cos \theta_{i k}-Q_{o k} \cos \theta_{o k}-\frac{M_{g k}}{D} \sin \theta_{i k}=0(34)$
$f_{4}=Q_{i k} \sin \theta_{i k}+\frac{M_{g k}}{D} \cos \theta_{i k}-Q_{o k} \sin \theta_{o k}-\frac{M_{g k}}{D} \cos \theta_{o k}=0(35)$

According to the geometrical relationship, equation $\mathrm{f}_{5}$ and $\mathrm{f}_{6}$ were obtainedl:

$f_{5}=B O^{\prime \prime} * \cos \theta_{\mathrm{o} k}+D O^{\prime \prime} * \cos \theta_{i k}-B D * \cos \alpha^{\prime}=0(36)$

$f_{6}=B O^{\prime \prime} * \sin \theta_{o k}+D O^{\prime \prime} * \sin \theta_{i k}-B D * \sin \alpha^{\prime}=0$ (37)

Where, $\theta_{\mathrm{ik}}$ and $\theta_{\mathrm{ok}}$ are contact angle of inner ring and outer ring, $\mathrm{Q}_{\mathrm{ik}}$ and $\mathrm{Q}_{\mathrm{ok}}$ are contact force of inner ring and outer ring, and $\mathrm{DO}^{\prime \prime}, \mathrm{BO}^{\prime \prime}$ can be expressed as:

$\mathrm{DO}^{\prime \prime}=\left(f_{i}-0.5\right) D_{w}+\delta_{i k}(38)$

$\mathrm{BO}^{\prime \prime}=\left(f_{e}-0.5\right) D_{w}+\delta_{o k}(39)$

Combined equation $\mathrm{f}_{1}$ to $\mathrm{f}_{6}$ in together, a nonlinear equations can be given, where $\mathrm{e}_{\mathrm{i}}, \mathrm{e}_{\mathrm{o}}, \theta_{\mathrm{ik}}, \theta_{\mathrm{ok}}$, $\mathrm{Q}_{\mathrm{ik}}, \mathrm{Q}_{\mathrm{ok}}$ are unknown parameters. According to Newton iterative method, the nonlinear equations can be solved.

$$
\left\{\begin{array}{c}
f_{1}=\frac{2\left(1-e_{i}^{2}\right)}{e_{i}^{2}} \frac{E\left(e_{i}\right)-K\left(e_{i}\right)}{E\left(e_{i}\right)}+1-\frac{B_{i}-A_{i}}{B_{i}+A_{i}} \\
f_{2}=\frac{2\left(1-e_{o}^{2}\right)}{e_{o}^{2}} \frac{E\left(e_{o}\right)-K\left(e_{o}\right)}{E\left(e_{o}\right)}+1-\frac{B_{o}-A_{o}}{B_{o}+A_{o}} \\
f_{3}=F_{c k}+\frac{M_{g k}}{D} \sin \theta_{o k}+Q_{i k} \cos \theta_{i k}-Q_{o k} \cos \theta_{o k}-\frac{M_{g k}}{D} \sin \theta_{i k} \\
f_{4}=Q_{i k} \sin \theta_{i k}+\frac{M_{g k}}{D} \cos \theta_{i k}-Q_{o k} \sin \theta_{o k}-\frac{M_{g k}}{D} \cos \theta_{o k} \\
f_{5}=B O^{\prime \prime} * \cos \theta_{i k}+D O^{\prime \prime} * \cos \theta_{i k}-B D * \cos \alpha^{\prime} \\
f_{6}=B O^{\prime \prime} * \sin \theta_{o k}+D O^{\prime \prime} * \sin \theta_{i k}-B D * \sin \alpha^{\prime}
\end{array}\right.
$$

The iterative method is described as follows:

$$
\mathrm{J}(\mathrm{x})=\left[\begin{array}{l}
\frac{\partial f_{1}}{\partial e_{i}} \frac{\partial f_{1}}{\partial e_{o}} \frac{\partial f_{1}}{\partial \theta_{i k}} \frac{\partial f_{1}}{\partial \theta_{o k}} \frac{\partial f_{1}}{\partial Q_{i k}} \frac{\partial f_{1}}{\partial Q_{o k}} \\
\frac{\partial f_{2}}{\partial e_{i}} \frac{\partial f_{2}}{\partial e_{o}} \frac{\partial f_{2}}{\partial \theta_{i k}} \frac{\partial f_{2}}{\partial \theta_{o k}} \frac{\partial f_{2}}{\partial Q_{i k}} \frac{\partial f_{2}}{\partial e_{o k}} \frac{\partial f_{3}}{\partial e_{o}} \frac{\partial f_{3}}{\partial \theta_{i k}} \frac{\partial f_{3}}{\partial \theta_{o k}} \frac{\partial f_{3}}{\partial Q_{i k}} \frac{\partial f_{3}}{\partial Q_{o k}} \\
\frac{\partial f_{4}}{\partial e_{i}} \frac{\partial f_{4}}{\partial e_{o}} \frac{\partial f_{4}}{\partial \theta_{i k}} \frac{\partial f_{4}}{\partial \theta_{o k}} \frac{\partial f_{4}}{\partial Q_{i k}} \frac{\partial f_{4}}{\partial Q_{o k}} \\
\frac{\partial f_{5}}{\partial e_{i}} \frac{\partial f_{5}}{\partial e_{o}} \frac{\partial f_{5}}{\partial \theta_{i k}} \frac{\partial f_{5}}{\partial \theta_{o k}} \frac{\partial f_{5}}{\partial Q_{i k}} \frac{\partial f_{5}}{\partial Q_{o k}} \\
\frac{\partial f_{6}}{\partial e_{i}} \frac{\partial f_{6}}{\partial e_{o}} \frac{\partial f_{6}}{\partial \theta_{i k}} \frac{\partial f_{6}}{\partial \theta_{o k}} \frac{\partial f_{6}}{\partial Q_{i k}} \frac{\partial f_{6}}{\partial Q_{o k}}
\end{array}\right]
$$

$\mathrm{J}\left(x^{(k)}\right) d^{(k)}=-F\left(x^{(k)}\right)(42)$

$x^{(k+1)}=x^{(k)}+d^{(k)}$ 